\title{
Circulating fatty acids and prostate cancer risk in a nested case- control study: the Multiethnic Cohort
}

\author{
Song-Yi Park and Lynne R. Wilkens \\ Epidemiology Program, Cancer Research Center of Hawaii, University of Hawaii, 1236 Lauhala \\ Street, Honolulu, HI 96813, USA
}

\section{Susanne M. Henning}

Center for Human Nutrition, David Geffen School of Medicine, University of California, Los Angeles, CA, USA

\author{
Loïc Le Marchand \\ Epidemiology Program, Cancer Research Center of Hawaii, University of Hawaii, 1236 Lauhala \\ Street, Honolulu, HI 96813, USA

\section{Kun Gao} \\ Center for Human Nutrition, David Geffen School of Medicine, University of California, Los Angeles, \\ CA, USA
}

\author{
Marc T. Goodman and Suzanne P. Murphy \\ Epidemiology Program, Cancer Research Center of Hawaii, University of Hawaii, 1236 Lauhala \\ Street, Honolulu, HI 96813, USA
}

\section{Brian E. Henderson}

Preventive Medicine, Keck School of Medicine, University of Southern California, Los Angeles, CA, USA

\section{Laurence N. Kolonel}

Epidemiology Program, Cancer Research Center of Hawaii, University of Hawaii, 1236 Lauhala Street, Honolulu, HI 96813, USA

\begin{abstract}
Objective-Dietary fat, including specific fatty acids, has been proposed to contribute to prostate cancer pathogenesis, but findings from the studies based on biomarkers have been conflicting.

Methods-We examined the association between erythrocyte membrane fatty acid composition and prostate cancer risk in a nested case-control study within a multiethnic cohort of African Americans, Native Hawaiians, Japanese Americans, Latinos, and Whites. Analyses included 376 cases and 729 matched controls. Conditional logistic regression was used to estimate the odds ratios and $95 \%$ confidence intervals with adjustment for multiple covariates.
\end{abstract}

Results-No significant association was found for saturated, mono- and polyunsaturated fatty acid, or for specific n-3 and n-6 fatty acids, even when the analysis was limited to advanced or high grade prostate cancer. In ethnic specific analyses, there was a positive association with palmitic acid in Japanese Americans that was significantly different from the null results in other groups. There was also an increased risk with n-3 fatty acids and the ratio of n-3/n-6 fatty acids in Whites.

\footnotetext{
(C) Springer Science+Business Media B.V. 2008

e-mail: spark@crch.hawaii.edu
} 
Conclusion-Although there was a suggestion of ethnic specific associations with some fatty acids, our overall findings do not support a role for fatty acids in prostate carcinogenesis.

\section{Keywords}

Prostatic neoplasms; Fatty acids; Erythrocytes; Nested case-control study

\section{Introduction}

Dietary fat, including specific fatty acids, has been proposed to contribute to prostate cancer pathogenesis [1-3]. While early studies suggested that total, saturated, or animal fat might increase prostate cancer risk, animal experiments and in vitro studies indicate that different types of fatty acids have opposing effects on the development and progression of prostate cancer: long chain $\mathrm{n}-3$ polyunsaturated fatty acids (PUFAs), such as eicosapentaenoic acid (EPA, C20:5 n-3) and docosahexaenoic acid (DHA, C22:6 n-3), suppress the development of cancer [4], whereas n-6 fatty acids including linoleic acid (C18:2 n-6) and arachidonic acid (C20:4 n-6) promote carcinogenesis [5]. Therefore, the ratio of n-3 to $n-6$ fatty acids may be more important than the absolute intake of $n-3$ fatty acids [5]. One of the possible mechanisms for the anticarcinogenic effects of $n-3$ fatty acids is the suppression of arachidonic acid-derived eicosanoid biosynthesis, which is involved in tumor growth [4]. A few observational studies have found that high intake of n-3 PUFA $\alpha$-linolenic acid (ALA, C18:3 n-3) increased prostate cancer risk, and have suggested interference with $5 \alpha$-reductase and formation of free radicals from fatty acid oxidation as possible mechanisms for the carcinogenic effect [6-8].

A number of epidemiologic studies have examined the intakes of specific fatty acids from foods in relation to prostate cancer risk, but not all have supported the suggested mechanisms or hypotheses [6-16]. Although not perfect biomarkers of dietary exposures, fatty acids in blood or tissue as quantitative indicators of dietary intake may provide additional insight for testing hypotheses about diet-disease relationships over self-reported dietary intake $[17,18]$.

Previously, we reported no association between the intake of dietary fatty acids and prostate cancer risk in the Multiethnic Cohort Study [19]. In order to further investigate this association based on biomarkers, we analyzed data from a nested case-control study within our cohort that measured fatty acid composition in erythrocyte membranes.

\section{Materials and methods}

\section{Study population}

The Multiethnic Cohort Study was established to test hypotheses related to diet and cancer in Hawaii and Los Angeles, California [20]. It was designed to include adults from five targeted racial/ethnic groups: African Americans, Native Hawaiians, Japanese Americans, Latinos, and Whites. In 1993-1996, more than 215,000 participants aged 45-75 years completed a 26-page questionnaire on diet, medical history, and lifestyle. All the baseline information from the cohort questionnaire was available for the current study. Information of particular potential value for the present study included weight, height, physical activity, education, and family history of prostate cancer.

\section{Collection and preparation of specimens}

Cohort members were recruited to supply prospective biospecimens; this recruitment largely took place between 2001 and 2006. Once the subjects gave informed consent to participate, a short screening questionnaire (use of anticoagulants, blood clotting disorders, etc.) and updated information on a few items (current smoking habits, weight, vitamin supplement use, etc.) was 
administered by phone. Blood collections were scheduled early in the morning so as to obtain fasting blood (minimum $10 \mathrm{~h}$ ). After centrifugation and separation into the four components (serum, plasma, buffy coat, and red cells), aliquots of each component were sealed in $0.5 \mathrm{cc}$ cryotubes using an automatic blood component dispensing machine. The specimens were stored in liquid nitrogen freezers. A total of 67,594 members contributed to the biorepository, of which 31,136 were men. When comparing the characteristics of individuals who provided specimens with those who did not, there was no substantial difference between the two groups in the family history of cancer, body mass index, fat and vegetable intake, and physical activity. Therefore, the biospecimen repository participants are broadly representative of all cohort members.

\section{Selection of cases and controls}

Identification of incident prostate cancer cases was accomplished through the tumor registries of the Surveillance, Epidemiology and End Results (SEER) Program of the National Cancer Institute covering Hawaii and California. Deaths were identified by linkage to death-certificate files in Hawaii and California and the National Death Index. For the nested case-control study of biomarkers and prostate cancer, we defined cases as men who contributed blood to the biorepository subcohort and who were diagnosed with invasive prostate cancer after blood collection up to the date of the latest tumor registry linkage in 2006; the average length between blood collection and cancer diagnosis for the cases included in the analyses was 1.9 years. Advanced prostate cancers were defined as all cancers that were regional or metastatic (not in situ or localized). High grade prostate cancers were based on Gleason score ( $\geq 7$ categorized as poorly differentiated).

Controls were selected from among the male participants who contributed blood to the biorepository. For each case, a pool was created including potential controls who were alive and free of prostate cancer at the age of case's diagnosis and who matched the case on geographic location (Hawaii or California), race/ethnicity, birth year ( \pm 1 year), date of blood draw ( \pm 6 months), time of blood draw ( $\pm 2 \mathrm{~h}$ ), and fasting hours ( 0 to $<6,6$ to $<8,8$ to $<10$, and $10+\mathrm{h})$. Two controls were randomly selected from each pool.

Of the 467 eligible prostate cancer cases, 77 cases were excluded from fatty acid level assessment because they did not have fasting blood samples. Additionally, 14 cases were not analyzed because of technical problems. Therefore, complete data for the present study was available for 376 cases (including 102 advanced or high grade cancer cases) and 729 matched controls.

\section{Laboratory analyses}

Erythrocyte membrane fatty acids were determined by the method of Bagga et al. [21]. Heparinized blood was centrifuged, and the plasma was removed. Approximately $4 \mathrm{ml}$ of the packed heparinized red cells were suspended in phosphate buffer saline (PBS) buffer, mixed, and centrifuged for five minutes. The supernatant was removed, and the wash was repeated a second time. After the second wash, the clear supernatant was again removed. A dilution of the red cells was made: $3 \mathrm{ml}$ of washed red blood cell to $1 \mathrm{ml}$ of PBS. This thick mixture was aspirated into the straws.

Samples were analyzed in duplicate. For the red blood cell membrane fatty acid analysis, samples were thawed, centrifuged at 2,900 $\mathrm{g}$ for $30 \mathrm{~min}$, and the pellet was used for fatty acid analysis. Total fatty acid was extracted and converted to fatty acid methyl esters (FAMEs) using methanol/benzene mixture (8:2, v:v). Heptadecanoic acid (C17:0) was used as the internal standard. The mixture was frozen at $-70^{\circ} \mathrm{C}$ for $20 \mathrm{~min}$ prior to the addition of acetylchloride. The mixture was heated at $100^{\circ} \mathrm{C}$ for $60 \mathrm{~min}$, then, cooled and neutralized with 
$6 \%$ potassium carbonate. Samples were centrifuged at 2,900 $g$ and the supernatant was injected into the gas chromatograph. FAME was separated and quantified by the use of Agilent Technologies (Wilmington, DE) 5890A series II gas chromatography fitted with a model 7673 automatic split-injection system and flame ionization detector and SP2380 stabilized phase fused silica capillary column $(30 \mathrm{~m} \times 0.32 \mathrm{~mm}$ i.d., $0.25 \mu \mathrm{m}$ film thickness, Supelco, Inc, Bellefonte, PA). Quantification was based on the recovery of a known quantity of the internal standard (C17:0, NuChek Preparation Inc., Elysian, MN), and on the response ratio of fatty acid standards purchased from NuChek Preparation Inc. (Elysian, MN). For quality control, we used a pooled serum sample which was analyzed with each batch of samples. Using this pooled serum sample, the following coefficients of variation have been established: C16:0 1.6\%, C16:1 2.7\%, C18:0 2.6\%, C18:1 3.4\%, C18:2 0.7\%, C18:3 0.7\%, C20:2 8.7\%, C20:4 $0.7 \%, \mathrm{C} 20: 53.2 \%, \mathrm{C} 22: 54.2 \%$, and $\mathrm{C} 22: 62.2 \%$.

FAMEs were identified by comparing with authentic FAME standards (Nu-Check Prep, Elysian, MN), and the peak areas were integrated as wt.\% with a dedicated microprocessor. Individual peaks were distinguished and measured, including those constituting as little as $0.05 \%$ of the FAME.

\section{Statistical analyses}

Each fatty acid was represented as the percentage of total fatty acids in the erythrocyte membranes. The distributions of fatty acids between cases and controls were compared. Odds ratios (ORs) and 95\% confidence intervals (95\% CIs) were calculated with conditional logistic regression, modeling cancer status. Fatty acid composition was divided into quartiles or tertiles, which was determined by the overall distribution of each fatty acid in both cases and controls. Trend tests were performed by entering a variable assigned the median of the appropriate quantile group as a continuous variable. $p$ values for dose-response were based on the Wald statistics for trend variables.

Matched sets were used as strata in the conditional logistic models, which accounted for the matching criteria: location, race/ethnicity, birth year, date of blood draw, time of blood draw, and fasting hours. The ORs were further adjusted for age at blood draw and fasting hours prior to blood draw as continuous variables to account for any possible systematic differences within matched sets, as well as family history of prostate cancer, BMI, and education, which were found to be related with prostate cancer risk in the full multiethnic cohort analysis. Other potential confounders such as physical activity and alcohol consumption were evaluated, but not included in the models because they did not alter the associations. The models were repeated separately for the four larger ethnic groups. Native Hawaiians were not included in these ethnicspecific analyses due to the small number of cases. Tests for the interaction between fatty acid composition and ethnicity in relation to prostate cancer risk were based on the likelihood ratios between models with and without interaction terms. Also, the models were run including only advanced or high grade tumors as cases and their matched controls. We repeated the analyses excluding controls with high prostate specific antigen (PSA) values $(>4.0 \mathrm{ng} / \mathrm{ml})$ in order to reduce the disease misclassification in the control group. In order to find out whether obesity and smoking status affect the association between fatty acid composition and prostate cancer risk, we performed the analyses stratified by BMI $(<25,25-30$, and $\geq 30)$ and smoking status (never, former, and current smokers). These analyses used unconditional logistic regression to maximize the number of subjects available and adjusting for matching criteria variables. Statistical significance was considered at $p<0.05$ and all tests were two sided.

\section{Results}

Compared to prostate cancer controls, cases were more likely to have a family history of prostate cancer (Table 1). There were no differences in educational level, BMI, physical 
activity, and alcohol consumption between cases and controls. Mean fatty acid composition in erythrocyte membranes did not differ between cases and controls either for individual fatty acids or for the ratios of n-3/n-6 or polyunsaturated/saturated fatty acids.

Table 2 presents the ORs for associations of fatty acid composition in erythrocyte membranes with prostate cancer risk. Neither saturated, monounsaturated nor polyunsaturated (n-3 or n-6) fatty acids as a group shows an association with prostate cancer. In addition, none of the 11 component fatty acids within these four groups show any association. Furthermore, the ratios of n-3 to n-6 fatty acids and polyunsaturated to saturated fatty acids show no association with prostate cancer risk. When the analysis was limited to advanced or high grade prostate cancer, the results were similar (Table 3 ). There were, however, two suggestive results that could be nonsignificant due to statistical power: that for EPA with ORs of 1.00, 1.44, and 1.61 ( $p$ for trend $=0.23$ ) and that for total PUFA with ORs of $1.00,1.08$, and 1.78 ( $p$ for trend $=0.22$ ).

For the ethnic-specific analyses, the sample sizes were limited and for Native Hawaiians in particular, they were too few to include. As shown in Table 4, a positive association with palmitic acid (C16:0) is seen among Japanese Americans (OR of the highest vs. the lowest tertile $=3.21,95 \% \mathrm{CI}=1.21-8.53, p$ for trend $=0.009)$, but not in any of the other ethnic groups $(p$ for interaction $=0.05$ ). Although the test for heterogeneity was not significant, in Whites, total $\mathrm{n}-3$ PUFA is also positively related to prostate cancer $(\mathrm{OR}=2.77,95 \% \mathrm{CI}=1.04$ $7.39, p$ for trend $=0.04, p$ for interaction $=0.12)$, as was the ratio of $n-3 / n-6$ fatty acids $(\mathrm{OR}=$ $2.92,95 \% \mathrm{CI}=1.01-8.47, p$ for trend $=0.04, p$ for interaction $=0.09$ ).

When the analyses were repeated excluding controls with high PSA values, the results did not change (data not shown). In an analysis stratified by BMI, the obese group (BMI $\geq 30$ ) showed an inverse association for prostate cancer risk with total saturated fatty acid and a positive association with total monounsaturated fatty acid (data not shown). However, the number of cases in each cell of the obese group was relatively small and the risk estimates uncertain. In an analysis stratified by smoking status, there were only a few statistically significant results with inconsistent patterns that may have been chance findings (data not shown).

\section{Discussion}

In this case-control study nested within the Multiethnic Cohort Study, we found no significant association between the fatty acid composition in erythrocyte membranes and the risk of total or advanced/high grade prostate cancer. In ethnic specific analyses, there was a positive association with palmitic acid in Japanese Americans that was significantly different from the null results in other groups. There was also an increased risk with $n-3$ fatty acids and the ratio of n-3/n- 6 fatty acids in Whites, but there was no statistically significant heterogeneity across the ethnic groups for these variables.

Many epidemiological studies have investigated the relationship between dietary intake of fat components, including specific fatty acids, and prostate cancer risk [22-27]. The results have been inconsistent generally. Although some investigators have reported inverse associations with EPA and DHA or their main food source, fish $[8,10,28]$, others did not $[29,30]$. Similarly, two meta-analyses found an association between ALA intake and increased risk of prostate cancer [31,32], yet the heterogeneity between the studies hampered the interpretation of the results [33].

We also investigated associations between fatty acid intake and prostate cancer risk in our cohort previously and found no relation in the overall analyses [19]. Since we and others have reviewed studies on dietary intake of fatty acids and prostate cancer [22,24,25,27], we focus here on investigations based on biomarkers of fatty acids. In the current study, we measured fatty acids in the membrane of red blood cells. An advantage of this tissue source over serum 
is that red cells have an average life span of 120 days, so these measurements reflect a reasonably long period of exposure. These biomarkers complement analysis of self-reported dietary data as they are not subject to recall bias.

We identified five prospective (nested case-control) [29,34-37] and three retrospective (casecontrol) [38-40] studies published since 1996 that examined fatty acids in whole blood, serum, erythrocyte membranes, or adipose tissue in relation to prostate cancer risk. Findings from these studies have been as conflicting as those based on self-reported fatty acid intake. Long chain n-3 PUFAs, such as EPA (C20:5) and DHA (C22:6), were inversely associated with prostate cancer risk in two studies [34,40], but not in the other studies [29,35,38,39]. ALA (C18:3 n-3) was also positively related to cancer in the two studies only [35,39]. Findings on linoleic acid (C18:2 n-6) were equally inconsistent, with inverse associations in three studies $[29,34,37]$ and positive associations in two studies $[38,39]$. No other $n-6$ fatty acid, including arachidonic acid, was related to prostate cancer risk in any of these studies.

One of the possible reasons that many studies failed to find an association between n-3 PUFAs and prostate cancer risk is a low range of dietary intake in the study populations [24]. EPA and DHA concentrations are lower in warm water fish than in cold water fish [24]. Indeed, median intake of n-3 PUFAs in male participants in the Multiethnic Cohort living in Hawaii and California was $1.8 \mathrm{~g} /$ day [19], which is relatively low when compared to $2.6 \mathrm{~g} /$ day among men in a prospective study in Sweden [16] where fatty fish consumption is greater than in the US [24]. However, mean compositions of these PUFAs in erythrocyte membranes among the current study participants were higher compared to other studies conducted in different areas in the US; mean EPA and DHA values in controls were $0.67 \%$ and $6.84 \%$ of total fatty acids, respectively in the current study, while $0.59 \%$ and $5.23 \%$ in a study in North Carolina [38], and $0.61 \%$ and $4.17 \%$ in Washington [39].

Only a few investigations have been conducted for the relation between saturated fatty acids in blood and prostate cancer risk. While the Physicians' Health Study found no association for saturated fatty acids [41], a nested case-control study in Norway showed a positive association for myristic acid (C14:0) and palmitic acid (C16:0) [35]. Also, the Alpha-Tocopherol, BetaCarotene Cancer Prevention Study observed a positive association for myristic acid (C14:0) [29]. In our study, we examined palmitic and stearic but not myristic acid. The only positive findings were for palmitic acid (C16:0) in Japanese Americans. Major food sources of palmitic acid (C16:0) are meat, fish, milk, and cheese [42]. However, in our previous papers, we found no association between consumption of these foods and prostate cancer risk in Japanese Americans, or in the overall cohort $[19,43]$.

Most participants in the previous biomarker studies were Whites. When the current analysis was restricted to Whites, the ratio of n-3/n-6 fatty acids was associated with an increased risk of prostate cancer. This finding is opposite to the hypothesis that $\mathrm{n}-3$ fatty acids may suppress and n-6 fatty acids may promote carcinogenesis [4]. However, heterogeneity across ethnic groups was not statistically significant, so these results may be due to chance, especially given the small number $(n=50)$ of cases among Whites.

There are several strengths to our study. The prospective design minimizes the possibility that the disease process affected fatty acid levels. In order to further confirm this, when we excluded controls with high PSA values, some of whom may have had prostate cancer, the results were unchanged. Erythrocyte membrane fatty acid composition is a valid biomarker of relative dietary levels of fatty acids and reflects usual diet over a period of months, while plasma or serum fatty acids reflect short term (hours) intake and adipose tissue reflects even longer term (years) intake $[18,44]$. Godley et al. [45] reported that fatty acids in erythrocyte membranes was a reasonable substitute for adipose tissue as a biomarker of essential fatty acid consumption 
given their reasonably high correlations with consumption over the previous year (correlation coefficient $=0.44$ for EPA and 0.41 for DHA), and the fact that adipose tissue is more difficult to obtain and analyze than blood. In the present study, fatty acid composition in erythrocyte membranes was significantly correlated with dietary intake measured by a quantitative food frequency questionnaire $(\mathrm{QFFQ}$ ) at baseline (correlation coefficient $=0.23$ for EPA and 0.39 for DHA, $p<0.001$ ), despite a substantial time gap between baseline and blood collection ( 6.8 \pm 2.3 years). Also, we had information on several potential confounding factors and were able to evaluate whether they affected the fatty acid-prostate cancer associations.

Although we examined the relationship of fatty acids and prostate cancer risk by tumor stage, ethnicity, BMI, and smoking status, the number of cases was not large enough to thoroughly assess the associations in subgroup analyses. In particular, EPA and total PUFA showed a nonsignificant positive trend for advanced or high grade prostate cancer. In the unconditional logistic models adjusting for matching criteria and using all controls to gain more power, ORs for EPA were 1.00, 1.15, and 1.36 ( $p$ for trend $=0.26$ ), which is still nonsignificant, while those for total PUFA were 1.00,1.44, and 2.51 with a significant positive trend ( $p$ for trend $<0.001$ ). Therefore, we cannot rule out a weak positive effect, especially for the trend of total PUFA.

Because the follow-up period in our study was relatively short (average follow-up period $=1.9$ years), it is possible that a longer follow-up could lead to a different conclusion. Since the follow-up period from the baseline QFFQ, where the dietary intake of fatty acids was assessed, was longer (average follow-up period $=8.7$ years), we combined the dietary and biomarker data to look at long-term effects indirectly. We defined high (above the median) dietary intake/ high biomarker, high intake/low (below the median) biomarker, low intake/high biomarker, and low intake/low biomarker groups. However, we did not see any difference in the risk of prostate cancer between the four groups: for DHA, the respective proportions of the four groups are $30.8 \%, 19.3 \%, 19.2 \%$, and $30.7 \%$, and the ORs of prostate cancer were $0.94(0.67-1.30)$, 0.96 (0.66-1.39), $0.88(0.60-1.28)$, and 1.00 (ref.), respectively. This provides some evidence that the null results would persist over a longer term.

Although there was a suggestion of ethnic specific associations with some fatty acids, our overall findings do not support a role for fatty acids in prostate carcinogenesis.

\section{Acknowledgment}

This study was supported in part by the National Cancer Institute grants P01 CA33619 and R37 CA54281, and by contract N01-PC-35137 from the National Institutes of Health, Department of Health and Human Services.

\section{References}

1. Kolonel LN, Nomura AM, Cooney RV. Dietary fat and prostate cancer: current status. J Natl Cancer Inst 1999;91:414-428. [PubMed: 10070940]

2. Kolonel LN. Fat, meat, and prostate cancer. Epidemiol Rev 2001;23:72-81. [PubMed: 11588857]

3. Fleshner N, Bagnell PS, Klotz L, Venkateswaran V. Dietary fat and prostate cancer. J Urol 2004;171:S19-S24. [PubMed: 14713748]

4. Larsson SC, Kumlin M, Ingelman-Sundberg M, Wolk A. Dietary long-chain n-3 fatty acids for the prevention of cancer: a review of potential mechanisms. Am J Clin Nutr 2004;79:935-945. [PubMed: 15159222]

5. Rose DP. Effects of dietary fatty acids on breast and prostate cancers: evidence from in vitro experiments and animal studies. Am J Clin Nutr 1997;66:1513S-1522S. [PubMed: 9394709]

6. De Stefani E, Deneo-Pellegrini H, Boffetta P, Ronco A, Mendilaharsu M. Alpha-linolenic acid and risk of prostate cancer: a case-control study in Uruguay. Cancer Epidemiol Biomark Prev 2000;9:335338. 
7. Ramon JM, Bou R, Romea S, et al. Dietary fat intake and prostate cancer risk: a case-control study in Spain. Cancer Causes Control 2000;11:679-685. [PubMed: 11065004]

8. Leitzmann MF, Stampfer MJ, Michaud DS, et al. Dietary intake of n-3 and n-6 fatty acids and the risk of prostate cancer. Am J Clin Nutr 2004;80:204-216. [PubMed: 15213050]

9. Kristal AR, Cohen JH, Qu P, Stanford JL. Associations of energy, fat, calcium, and vitamin D with prostate cancer risk. Cancer Epidemiol Biomarkers Prev 2002;11:719-725. [PubMed: 12163324]

10. Augustsson K, Michaud DS, Rimm EB, et al. A prospective study of intake of fish and marine fatty acids and prostate cancer. Cancer Epidemiol Biomark Prev 2003;12:64-67.

11. Bidoli E, Talamini R, Bosetti C, et al. Macronutrients, fatty acids, cholesterol and prostate cancer risk. Ann Oncol 2005;16:152-157. [PubMed: 15598953]

12. Koralek DO, Peters U, Andriole G, et al. A prospective study of dietary alpha-linolenic acid and the risk of prostate cancer (United States). Cancer Causes Control 2006;17:783-791. [PubMed: 16783606]

13. Neuhouser ML, Barnett MJ, Kristal AR, et al. (n-6) PUFA increase and dairy foods decrease prostate cancer risk in heavy smokers. J Nutr 2007;137:1821-1827. [PubMed: 17585037]

14. Nagata Y, Sonoda T, Mori M, et al. Dietary isoflavones may protect against prostate cancer in Japanese men. J Nutr 2007;137:1974-1979. [PubMed: 17634273]

15. Liu X, Schumacher FR, Plummer SJ, et al. Trans-fatty acid intake and increased risk of advanced prostate cancer: modification by RNASEL R462Q variant. Carcinogenesis 2007;28:1232-1236. [PubMed: 17234723]

16. Wallstrom P, Bjartell A, Gullberg B, Olsson H, Wirfalt E. A prospective study on dietary fat and incidence of prostate cancer (Malmö, Sweden). Cancer Causes Control 2007;18:1107-1121. [PubMed: 17726648]

17. Willett, WC. Nutritional epidemiology. Oxford University Press; New York: 1998. p. 217-226.

18. Arab L, Akbar J. Biomarkers and the measurement of fatty acids. Public Health Nutr 2002;5:865871. [PubMed: 12638594]

19. Park SY, Murphy SP, Wilkens LR, Henderson BE, Kolonel LN. Fat and meat intake and prostate cancer risk: the multi-ethnic cohort study. Int J Cancer 2007;121:1339-1345. [PubMed: 17487838]

20. Kolonel LN, Henderson BE, Hankin JH, et al. A multi-ethnic cohort in Hawaii and Los Angeles: baseline characteristics. Am J Epidemiol 2000;151:346-357. [PubMed: 10695593]

21. Bagga D, Capone S, Wang HJ, et al. Dietary modulation of omega-3/omega-6 polyunsaturated fatty acid ratios in patients with breast cancer. J Natl Cancer Inst 1997;89:1123-1131. [PubMed: 9262250]

22. Zock PL, Katan MB. Linoleic acid intake and cancer risk: a review and meta-analysis. Am J Clin Nutr 1998;68:142-153. [PubMed: 9665108]

23. Pandian SS, Eremin OE, McClinton S, Wahle KW, Heys SD. Fatty acids and prostate cancer: current status and future challenges. J R Coll Surg Edinb 1999;44:352-361. [PubMed: 10612956]

24. Terry PD, Rohan TE, Wolk A. Intakes of fish and marine fatty acids and the risks of cancers of the breast and prostate and of other hormone-related cancers: a review of the epidemiologic evidence. Am J Clin Nutr 2003;77:532-543. [PubMed: 12600840]

25. Terry PD, Terry JB, Rohan TE. Long-chain (n-3) fatty acid intake and risk of cancers of the breast and the prostate: recent epidemiological studies, biological mechanisms, and directions for future research. J Nutr 2004;134:3412S-3420S. [PubMed: 15570047]

26. Astorg P. Dietary N-6 and N-3 polyunsaturated fatty acids and prostate cancer risk: a review of epidemiological and experimental evidence. Cancer Causes Control 2004;15:367-386. [PubMed: 15141138]

27. MacLean CH, Newberry SJ, Mojica WA, et al. Effects of omega-3 fatty acids on cancer risk: a systematic review. JAMA 2006;295:403-415. [PubMed: 16434631]

28. Terry P, Lichtenstein P, Feychting M, Ahlbom A, Wolk A. Fatty fish consumption and risk of prostate cancer. Lancet 2001;357:1764-1766. [PubMed: 11403817]

29. Mannisto S, Pietinen P, Virtanen MJ, et al. Fatty acids and risk of prostate cancer in a nested casecontrol study in male smokers. Cancer Epidemiol Biomark Prev 2003;12:1422-1428.

30. Allen NE, Sauvaget C, Roddam AW, et al. A prospective study of diet and prostate cancer in Japanese men. Cancer Causes Control 2004;15:911-920. [PubMed: 15577293] 
31. Brouwer IA, Katan MB, Zock PL. Dietary alpha-linolenic acid is associated with reduced risk of fatal coronary heart disease, but increased prostate cancer risk: a meta-analysis. J Nutr 2004;134:919-922. [PubMed: 15051847]

32. Dennis LK, Snetselaar LG, Smith BJ, Stewart RE, Robbins ME. Problems with the assessment of dietary fat in prostate cancer studies. Am J Epidemiol 2004;160:436-444. [PubMed: 15321840]

33. Attar-Bashi NM, Frauman AG, Sinclair AJ. Alpha-linolenic acid and the risk of prostate cancer. What is the evidence? J Urol 2004;171:1402-1407. [PubMed: 15017185]

34. Chavarro JE, Stampfer MJ, Li H, et al. A prospective study of polyunsaturated fatty acid levels in blood and prostate cancer risk. Cancer Epidemiol Biomark Prev 2007;16:1364-1370.

35. Harvei S, Bjerve KS, Tretli S, et al. Prediagnostic level of fatty acids in serum phospholipids: omega-3 and omega-6 fatty acids and the risk of prostate cancer. Int J Cancer 1997;71:545-551. [PubMed: 9178806]

36. King IB, Kristal AR, Schaffer S, Thornquist M, Goodman GE. Serum trans-fatty acids are associated with risk of prostate cancer in beta-carotene and retinol efficacy trial. Cancer Epidemiol Biomark Prev 2005;14:988-992.

37. Laaksonen DE, Laukkanen JA, Niskanen L, et al. Serum linoleic and total polyunsaturated fatty acids in relation to prostate and other cancers: a population-based cohort study. Int J Cancer 2004;111:444450. [PubMed: 15221975]

38. Godley PA, Campbell MK, Gallagher P, et al. Biomarkers of essential fatty acid consumption and risk of prostatic carcinoma. Cancer Epidemiol Biomark Prev 1996;5:889-895.

39. Newcomer LM, King IB, Wicklund KG, Stanford JL. The association of fatty acids with prostate cancer risk. Prostate 2001;47:262-268. [PubMed: 11398173]

40. Norrish AE, Skeaff CM, Arribas GL, Sharpe SJ, Jackson RT. Prostate cancer risk and consumption of fish oils: a dietary biomarker-based case-control study. Br J Cancer 1999;81:1238-1242. [PubMed: 10584888]

41. Gann PH, Hennekens CH, Sacks FM, et al. Prospective study of plasma fatty acids and risk of prostate cancer. J Natl Cancer Inst 1994;86:281-286. [PubMed: 8158682]

42. Jonnalagadda SS, Egan SK, Heimbach JT, Harris SS, Krisetherton PM. Fatty acid consumption pattern of Americans: 1987-1988 USDA Nationwide Food-Consumption Survey. Nutr Res 1995;15:17671781.

43. Park SY, Murphy SP, Wilkens LR, et al. Calcium, vitamin D, and dairy product intake and prostate cancer risk: The multi-ethnic cohort study. Am J Epidemiol 2007;166:1259-1269. [PubMed: 17925283]

44. Fuhrman BJ, Barba M, Krogh V, et al. Erythrocyte membrane phospholipid composition as a biomarker of dietary fat. Ann Nutr Metab 2006;50:95-102. [PubMed: 16373991]

45. Godley PA, Campbell MK, Miller C, et al. Correlation between biomarkers of omega-3 fatty acid consumption and questionnaire data in African American and Caucasian United States males with and without prostatic carcinoma. Cancer Epidemiol Biomark Prev 1996;5:115-119. 
Table 1

Characteristics of prostate cancer cases and controls

\begin{tabular}{|c|c|c|}
\hline & Cases & Controls \\
\hline Number of participants & 376 & 729 \\
\hline Age at blood draw (years) ${ }^{a}$ & $68.6 \pm 7.1^{b}$ & $68.5 \pm 7.2$ \\
\hline Fasting hours prior to blood draw ${ }^{c}$ & $13.5 \pm 2.9$ & $13.6 \pm 3.0$ \\
\hline Years of education & $13.8 \pm 2.9$ & $13.8 \pm 2.9$ \\
\hline Body mass index $\left(\mathrm{kg} / \mathrm{m}^{2}\right)$ & $26.1 \pm 4.0$ & $26.4 \pm 4.2$ \\
\hline METS of activity per day & $1.6 \pm 0.3$ & $1.6 \pm 0.3$ \\
\hline Alcohol consumption ( $\%$ total energy) & $3.9 \pm 7.5$ & $4.0 \pm 7.3$ \\
\hline Family history of prostate cancer (\%) & 12.5 & 7.1 \\
\hline \multicolumn{3}{|l|}{ Ethnicity $(\%)^{d}$} \\
\hline African Americans & 43.1 & 42.9 \\
\hline Native Hawaiians & 3.5 & 3.3 \\
\hline Japanese Americans & 21.3 & 21.5 \\
\hline Latinos & 18.9 & 18.7 \\
\hline Whites & 13.3 & 13.6 \\
\hline \multicolumn{3}{|l|}{ Fatty acids in erythrocyte membranes ( $\%$ total fatty acids) } \\
\hline Palmitic acid, C16:0 & $29.87 \pm 2.36$ & $29.81 \pm 1.78$ \\
\hline Palmitoleic acid, C16:1 & $0.49 \pm 0.54$ & $0.51 \pm 1.17$ \\
\hline Stearic acid, C18:0 & $21.95 \pm 1.80$ & $22.07 \pm 1.79$ \\
\hline Oleic acid, C18:1 & $16.41 \pm 2.25$ & $16.36 \pm 2.11$ \\
\hline Linoleic acid, C18:2 n-6 & $12.24 \pm 1.77$ & $12.29 \pm 1.74$ \\
\hline$\alpha$-Linolenic acid, C18:3 n-3 & $0.58 \pm 0.51$ & $0.55 \pm 0.40$ \\
\hline Eicosadienoic acid, C20:2 n-6 & $0.42 \pm 0.31$ & $0.43 \pm 0.34$ \\
\hline Arachidonic acid, C20:4 n-6 & $8.82 \pm 1.43$ & $8.88 \pm 1.22$ \\
\hline Eicosapentaenoic acid, C20:5 n-3 & $0.70 \pm 0.48$ & $0.67 \pm 0.53$ \\
\hline Docosapentanoic acid, C22:5 n-3 & $1.60 \pm 0.48$ & $1.60 \pm 0.48$ \\
\hline Docosahexaenoic acid, C22:6 n-3 & $6.93 \pm 2.02$ & $6.84 \pm 1.91$ \\
\hline n-3 fatty acids & $9.80 \pm 2.50$ & $9.66 \pm 2.42$ \\
\hline n-6 fatty acids & $21.48 \pm 2.25$ & $21.60 \pm 2.07$ \\
\hline $\mathrm{n}-3 / \mathrm{n}-6$ ratio & $0.47 \pm 0.15$ & $0.46 \pm 0.15$ \\
\hline Polyunsaturated fatty acid (PUFA) & $31.28 \pm 2.54$ & $31.25 \pm 2.36$ \\
\hline $\begin{array}{l}\text { Monounsaturated fatty acid } \\
\text { (MUFA) }\end{array}$ & $16.90 \pm 2.31$ & $16.86 \pm 2.31$ \\
\hline Saturated fatty acid (SFA) & $51.82 \pm 2.69$ & $51.88 \pm 2.53$ \\
\hline PUFA/SFA ratio & $0.61 \pm 0.08$ & $0.61 \pm 0.07$ \\
\hline
\end{tabular}

\footnotetext{
${ }^{a}$ Age at blood draw was matched within \pm 1 year

${ }^{b}$ Mean $\pm \mathrm{SD}$

${ }^{c}$ Fasting hours prior to blood draw was matched within the following categories: 0 to $<6,6$ to $<8,8$ to $<10$, and $10+\mathrm{h}$

$d_{\text {Ethnicity was a matching criterion }}$
}

Cancer Causes Control. Author manuscript; available in PMC 2010 March 1. 
Park et al.

Z倍

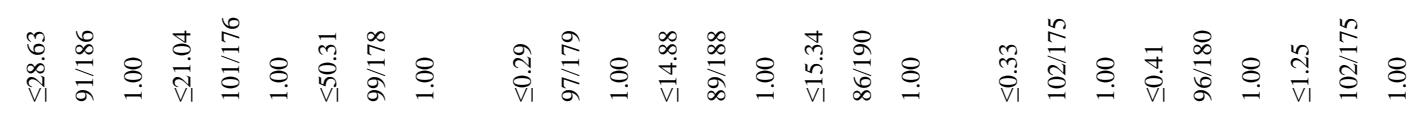

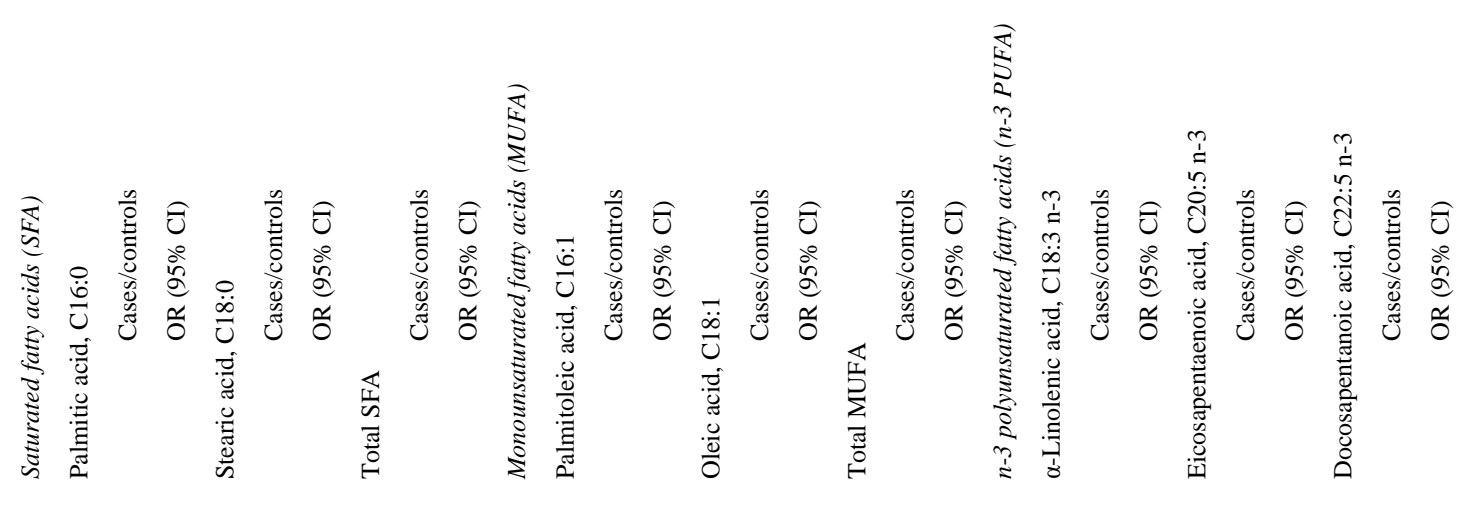




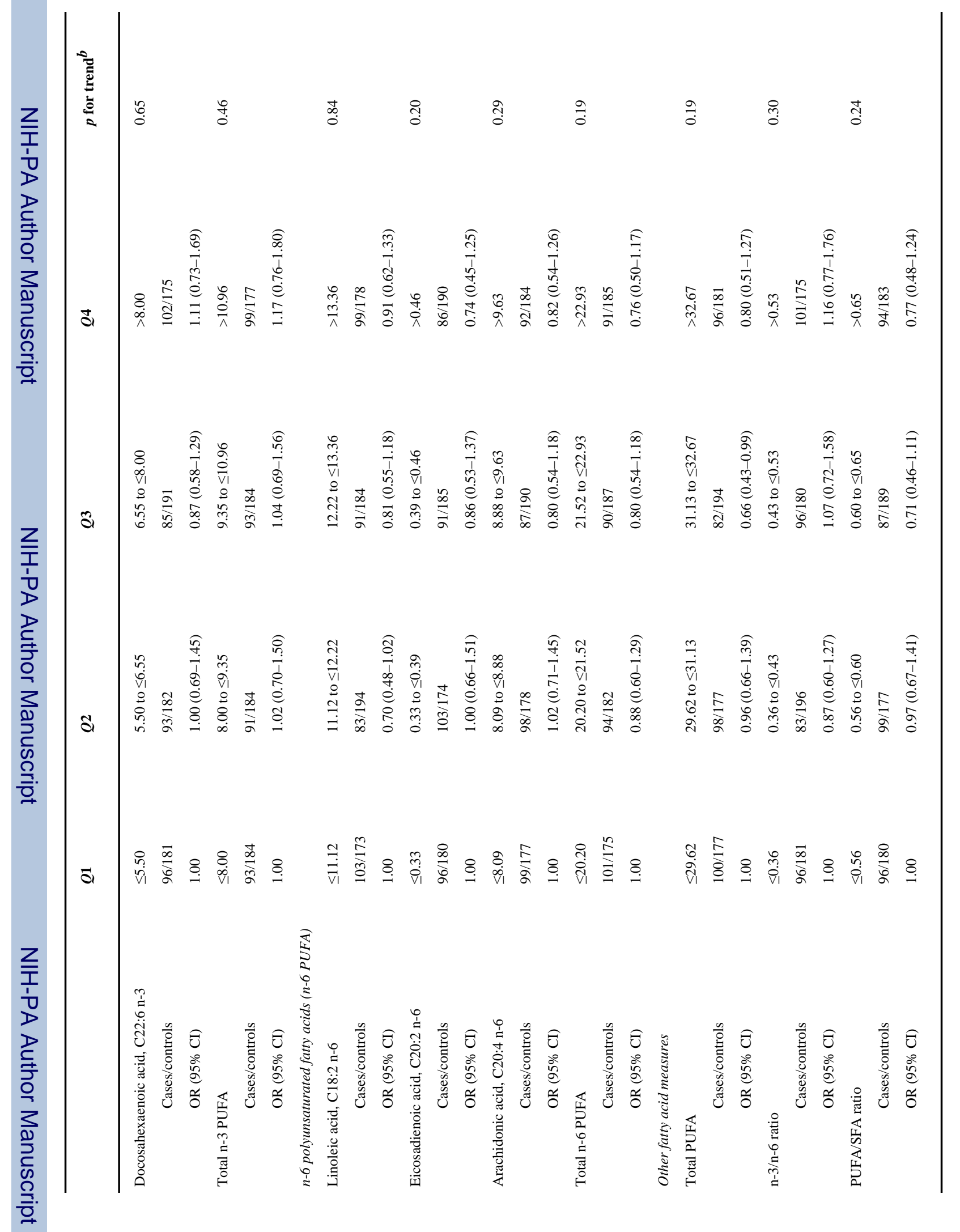




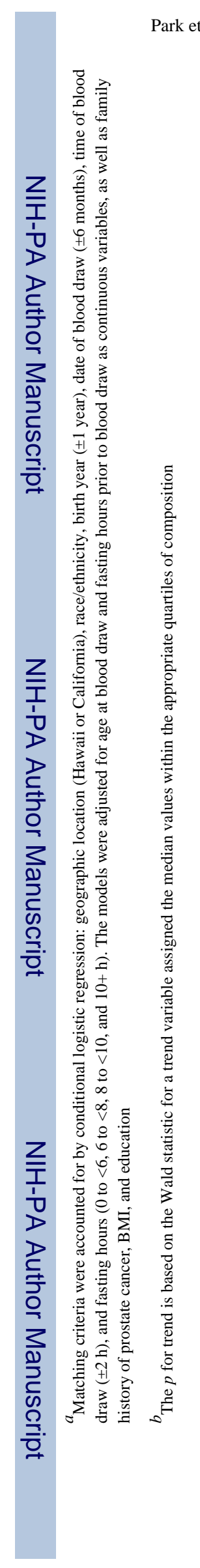

Cancer Causes Control. Author manuscript; available in PMC 2010 March 1. 


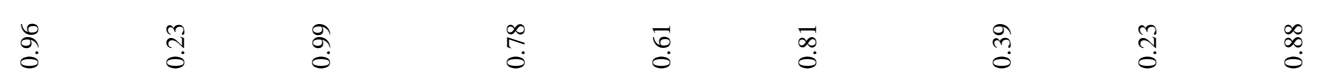

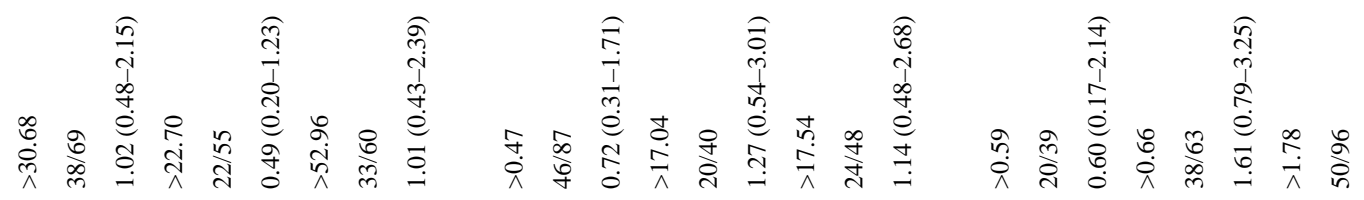

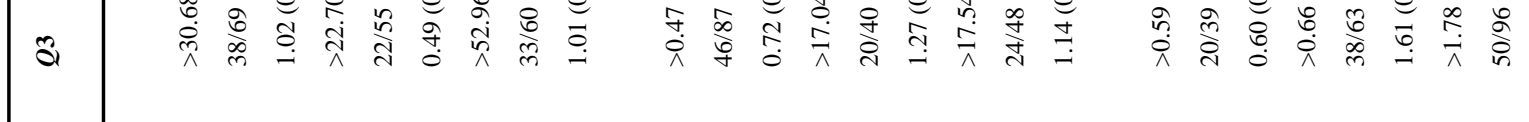




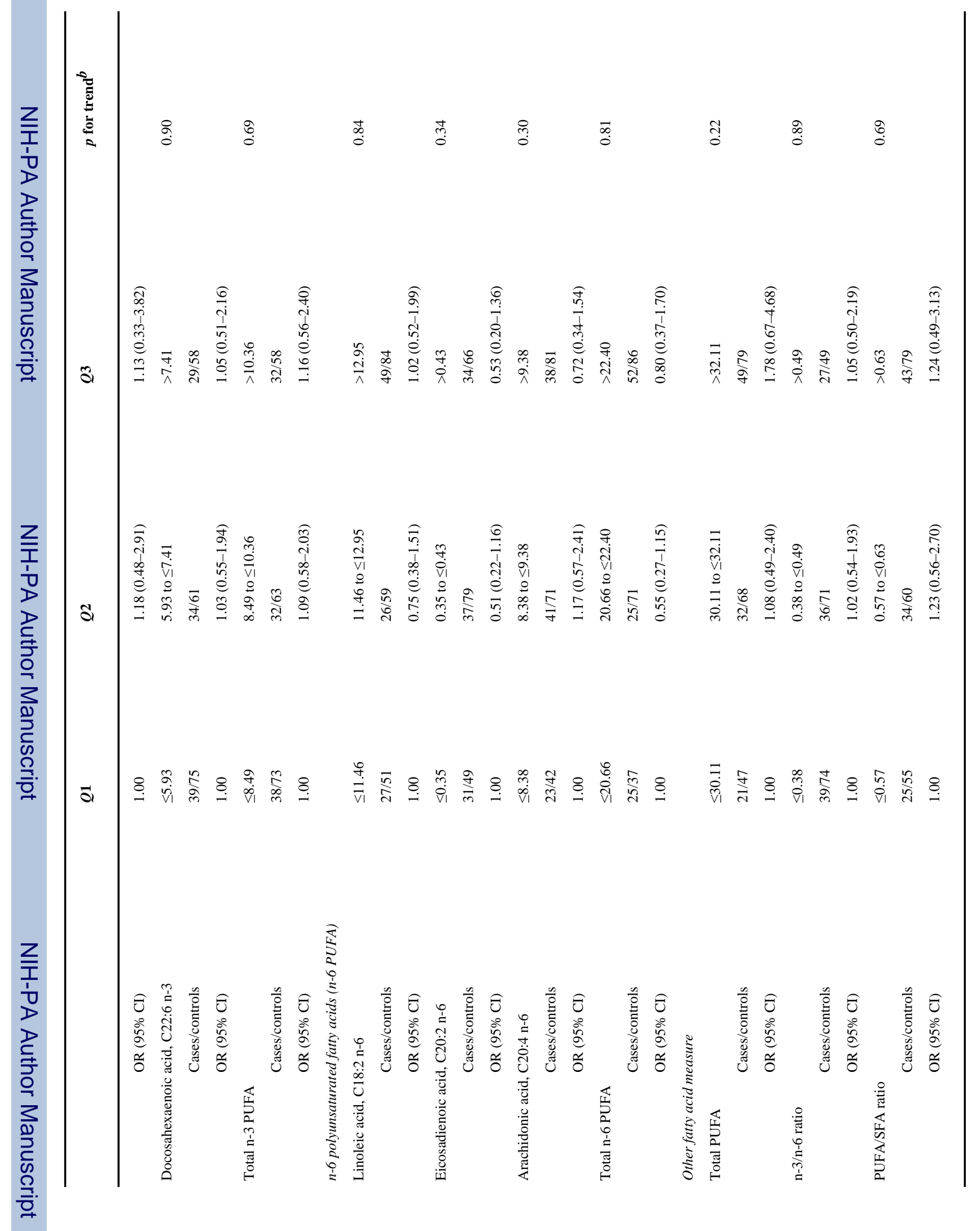




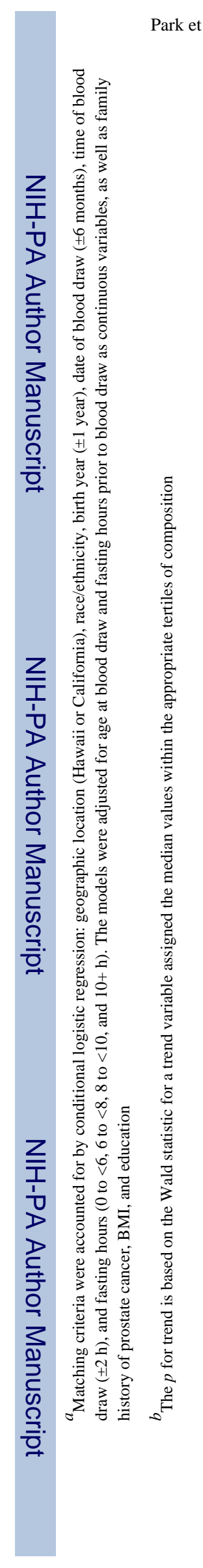

Cancer Causes Control. Author manuscript; available in PMC 2010 March 1. 


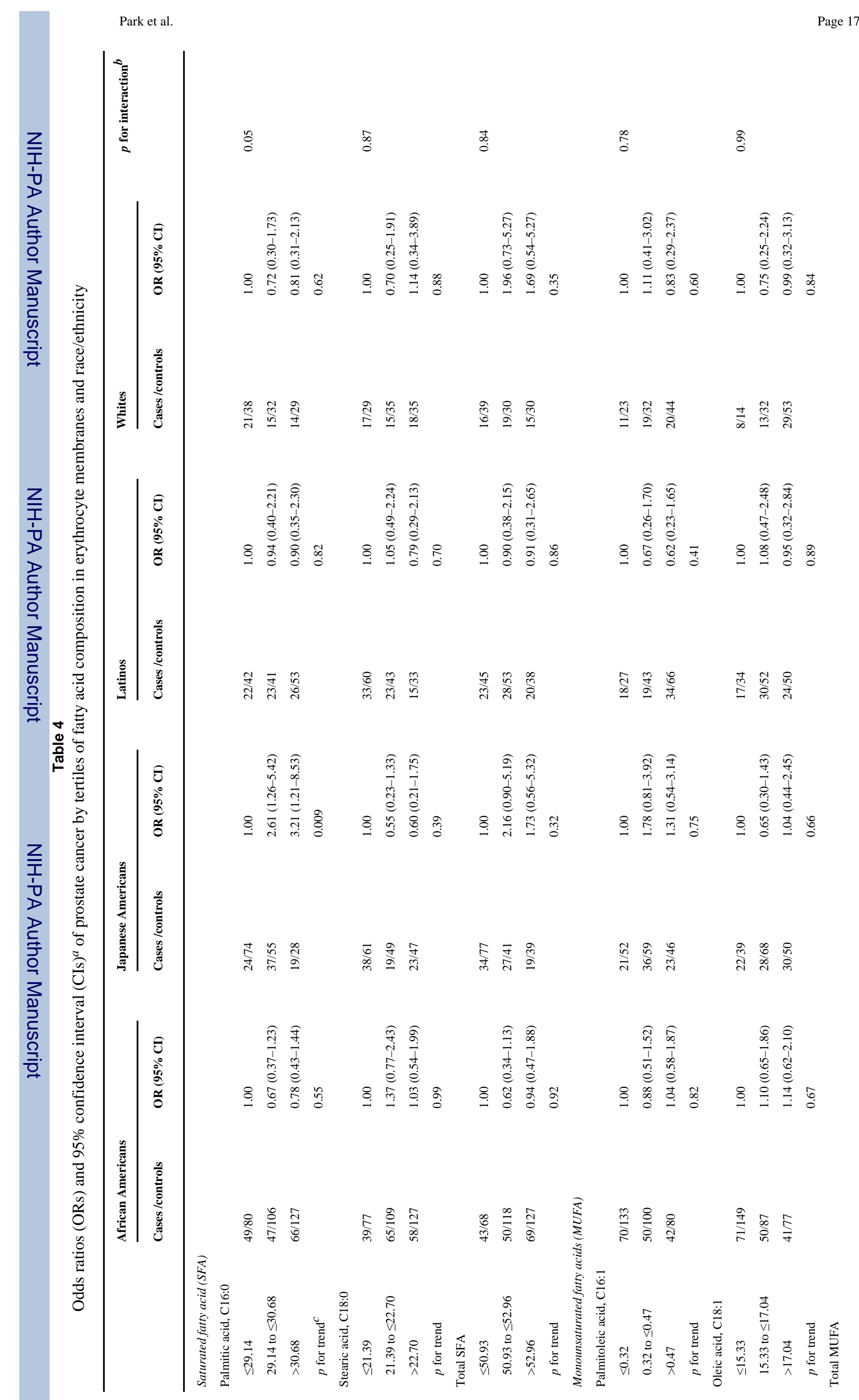




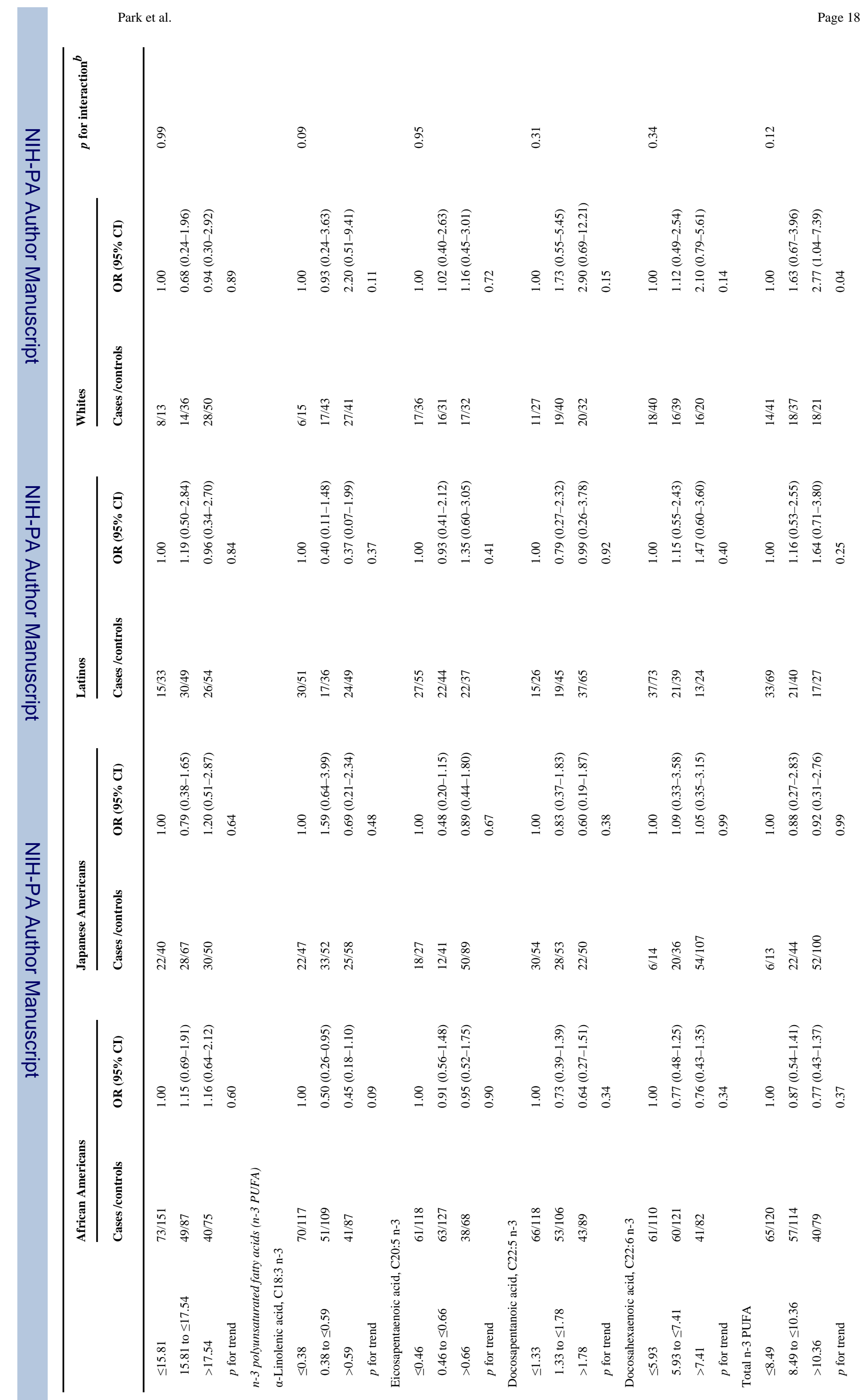




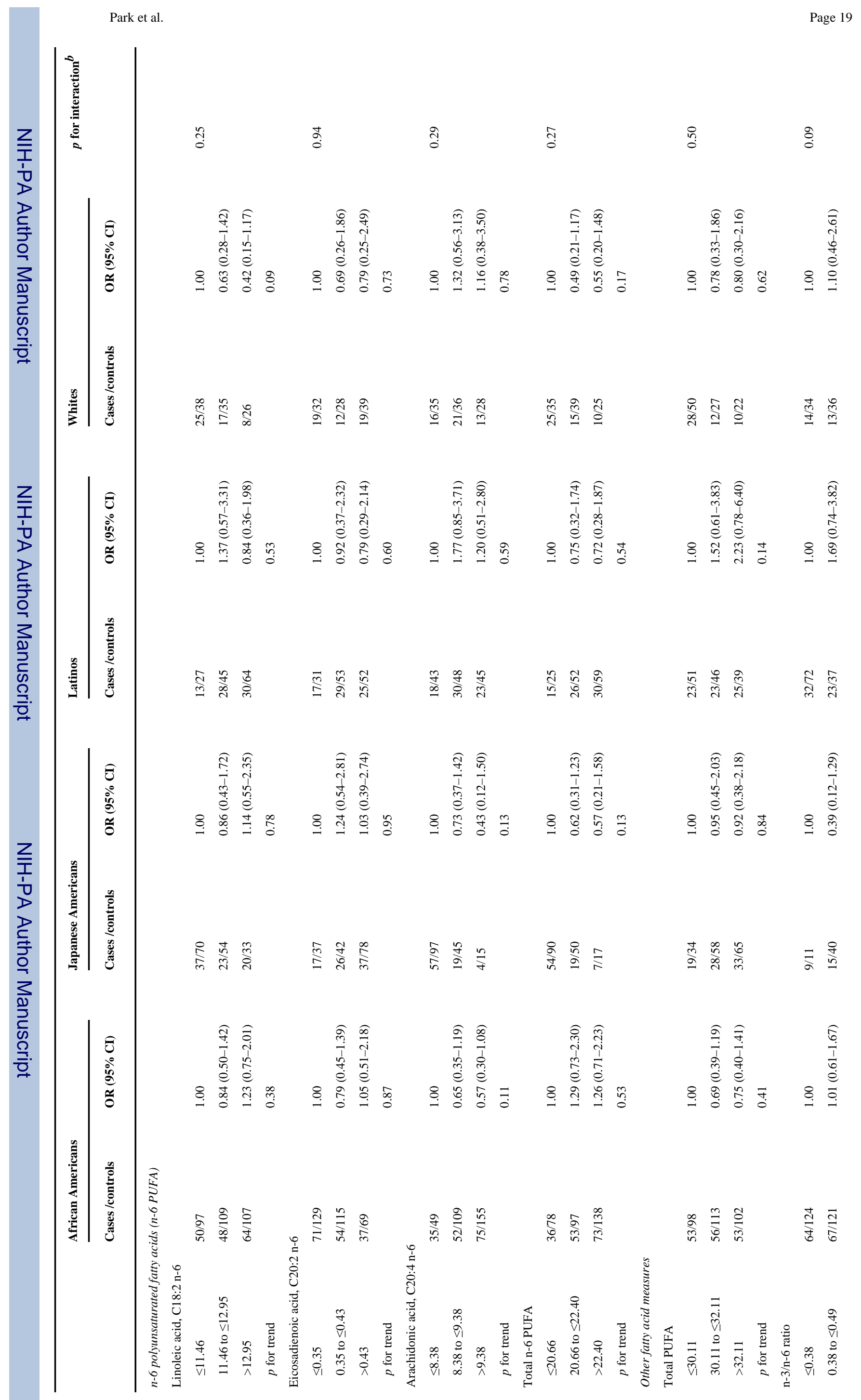




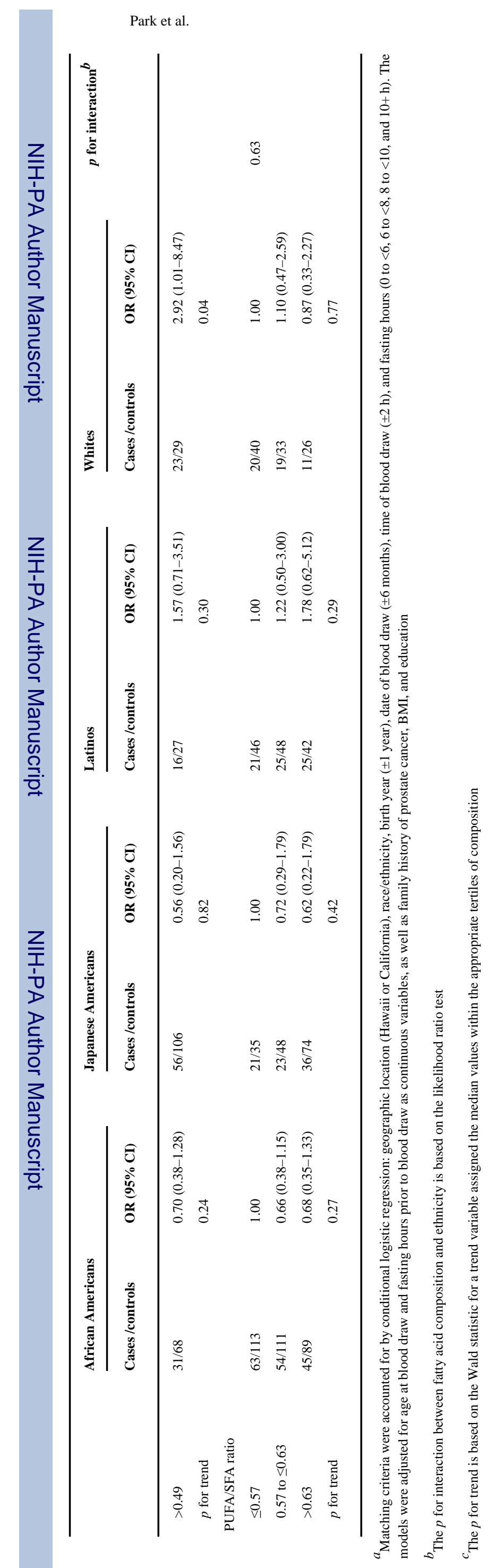

Page 20 